\title{
Eggshell and Feed Microbiota Do Not Represent Major Sources of Gut Anaerobes for Chickens in Commercial Production
}

\author{
Jiri Volf ${ }^{1}$, Magdalena Crhanova ${ }^{1}$, Daniela Karasova ${ }^{1}$, Marcela Faldynova ${ }^{1}$, Tereza Kubasova ${ }^{1}$, \\ Zuzana Seidlerova ${ }^{1}$, Alena Sebkova ${ }^{1}$, Michal Zeman ${ }^{1}{ }^{1}$, Helena Juricova ${ }^{1}$, Jitka Matiasovicova ${ }^{1}$, Marian Foltyn ${ }^{2}$, \\ Zdenek Tvrdon ${ }^{2}$ and Ivan Rychlik ${ }^{1, *}$
}

1 Veterinary Research Institute, Hudcova 70, 62100 Brno, Czech Republic; volf@vri.cz (J.V.); crhanova@vri.cz (M.C.); karasova@vri.cz (D.K.); faldynova@vri.cz (M.F.); kubasova@vri.cz (T.K.); seidlerova@vri.cz (Z.S.); asebkova@vri.cz (A.S.); zeman@vri.cz (M.Z.); juricova@vri.cz (H.J.); matiasovicova@vri.cz (J.M.)

2 Hatchery Vodnanske Kure, Komenskeho 75, 76811 Chropyne, Czech Republic; marian.foltyn@vodnanskekure.cz (M.F.); zdenek.tvrdon@vodnanskekure.cz (Z.T.)

* Correspondence: rychlik@vri.cz; Tel.: +420-533331201

\section{check for} updates

Citation: Volf, J.; Crhanova, M.; Karasova, D.; Faldynova, M.;

Kubasova, T.; Seidlerova, Z.; Sebkova,

A.; Zeman, M.; Juricova, H.;

Matiasovicova, J.; et al. Eggshell and Feed Microbiota Do Not Represent Major Sources of Gut Anaerobes for Chickens in Commercial Production. Microorganisms 2021, 9, 1480. https:// doi.org/10.3390/microorganisms 9071480

Academic Editor: Jana Seifert

Received: 19 June 2021

Accepted: 8 July 2021

Published: 11 July 2021

Publisher's Note: MDPI stays neutral with regard to jurisdictional claims in published maps and institutional affiliations.

Copyright: (c) 2021 by the authors. Licensee MDPI, Basel, Switzerland. This article is an open access article distributed under the terms and conditions of the Creative Commons Attribution (CC BY) license (https:// creativecommons.org/licenses/by/ $4.0 /)$.

\begin{abstract}
In this study, we addressed the origin of chicken gut microbiota in commercial production by a comparison of eggshell and feed microbiota with caecal microbiota of 7-day-old chickens, using microbiota analysis by $16 \mathrm{~S}$ rRNA sequencing. In addition, we tested at which timepoint during prenatal or neonatal development it is possible to successfully administer probiotics. We found that eggshell microbiota was a combination of environmental and adult hen gut microbiota but was completely different from caecal microbiota of 7-day-old chicks. Similarly, we observed that the composition of feed microbiota was different from caecal microbiota. Neither eggshell nor feed acted as an important source of gut microbiota for the chickens in commercial production. Following the experimental administration of potential probiotics, we found that chickens can be colonised only when already hatched and active. Spraying of eggs with gut anaerobes during egg incubation or hatching itself did not result in effective chicken colonisation. Such conclusions should be considered when selecting and administering probiotics to chickens in hatcheries. Eggshells, feed or drinking water do not act as major sources of gut microbiota. Newly hatched chickens must be colonised from additional sources, such as air dust with spores of Clostridiales. The natural colonisation starts only when chickens are already hatched, as spraying of eggs or even chickens at the very beginning of the hatching process did not result in efficient colonisation.
\end{abstract}

Keywords: chicken; microbiota; caecum; eggshell; hatchery; feed

\section{Introduction}

Birds or mammals are hatched or born nearly sterile, but immediately after birth they are exposed to an external environment, rich in different forms of life. Interactions between ubiquitously present prokaryotes and new-borns are frequent. Age-dependent colonisation of different animal body compartments by prokaryotic microbiota has been repeatedly described, including chickens and the chicken intestinal tract [1-4]. The intestinal tract of chickens is first colonised by E. coli, which is characteristic for chicken gut microbiota during the first week of life. E. coli is replaced by bacteria from phylum Firmicutes during the second week of life, mostly by aerotolerant Lactobacillaceae and spore-forming isolates from the Lachnospiraceae, Ruminococcaceae and Clostridiaceae families. Representatives of phylum Bacteroidetes and strict anaerobes from phylum Proteobacteria are usually among the last ones to appear in gut microbiota of chickens in commercial production. A commonly overlooked fact is that, due to the absence of hens in egg incubation and hatching, the development of gut microbiota in chickens in commercial production is highly specific, resulting in microbiota found in the caecum of one-week-old chickens originating 
exclusively from the environment $[4,5]$. If adult hens were present, the developmental pattern would be different [6]. However, if hens are excluded from this process, what is the origin bacterial species colonising chicken caecum in the first weeks of life?

This topic was addressed in the current study. Specifically, we asked two questions. Firstly, considering that the transmission of microbiota from hens to offspring is interrupted in commercial production, where do members of Lachnospiraceae or Ruminococcaceae families come from, and why do they appear in the chicken intestinal tract so shortly after hatching? Do they originate from eggshells, feed or drinking water? Secondly, at what timepoint during prenatal or neonatal development is it possible to colonise the chicken intestinal tract? Answers to these questions are of utmost importance because the rapid establishment of complex gut microbiota is crucial for chicken resistance to enteric infections [7-9]. To address these questions, we compared eggshell microbiota with gut microbiota of 7-day-old chicks. Next, we tested whether similar or different gut microbiota would develop in chickens hatched from eggs originating from different parent flocks, i.e., with different microbiota deposited on eggshells. We also investigated to what extent feed and drinking water act as a source of gut microbiota. Finally, we tested at what timepoint during egg incubation or hatching it is possible to administer bacterial strains to achieve successful gut colonisation.

\section{Materials and Methods}

\subsection{Comparison of Eggshell Microbiota with Caecal Microbiota of 7-Day-Old Chicks}

Forty-five fertilised eggs of ISA Brown egg laying line were obtained from a local hatchery. These eggs originated from 3 different reproductive flocks from different geographical locations in the Czech Republic (15 eggs per flock). Three eggs from each batch were used for DNA purification from eggshells and the remaining 12 eggs from each flock were incubated in a small-scale hatching incubator. Since the hatching efficiency in the small-scale experiment was around $50 \%$, five, five and four chickens were obtained from each parent flock, respectively. The chickens were labelled by leg rings and raised in the same cage. The chickens were sacrificed at the age of one week and caecal contents were collected for microbiota characterisation by sequencing the V3-V4 variable region of $16 \mathrm{~S}$ rRNA genes.

\subsection{Housing Conditions}

Chickens were kept in fully air-conditioned barrier rooms, in plastic boxes with slatted floors. Feed and drinking water were supplied ad libitum. Temperature was set to $34^{\circ} \mathrm{C}$ on day 1 ; this decreased by $2{ }^{\circ} \mathrm{C}$ per day. In addition, the boxes were equipped with brooders. A light regime started with $24 \mathrm{~h}$ light on day 1 , followed by $22 \mathrm{~h}$ light and $2 \mathrm{~h}$ dark on day $2,20+4$ h on day $3,19+5$ h on day 4 , and $18+6$ h on day 5 , and so on.

\subsection{DNA Purification from Eggshells}

Three visually clean eggs from each farm were used for DNA purification from eggshells according to [10], as follows: The inner and outer shell membranes were removed, and $50 \mathrm{mg}$ of eggshell was placed in a $2 \mathrm{~mL}$ tube with $2.5 \mathrm{~mm}$ zirconium silicate beads and $1600 \mu \mathrm{L}$ of 0.5 M EDTA. The shells were disrupted in a MagnaLyser (Roche, Basel, Switzerland) at $7000 \mathrm{rpm}$ for $60 \mathrm{~s}$, followed by overnight incubation on a shaker heated to $44^{\circ} \mathrm{C}$. Finally, DNA was purified from the supernatant using the DNeasy tissue kit and following the instructions of the manufacturer (Qiagen, Hilden Germany).

\subsection{Feed as a Source of Gut Microbiota}

Two groups of ISA Brown chickens with 4 birds per group were formed (different from the chickens used above for comparison of caecal and eggshell microbiota). The control group was provided with conventional feed and water throughout the whole experiment. The experimental group was provided with a fresh batch of autoclaved $\left(121^{\circ} \mathrm{C}\right.$ for $20 \mathrm{~min}$ ) feed and water on a daily basis. The two groups of chickens were kept 
in the same room, but in two different cages separated by $2 \mathrm{~m}$ distance to minimise any group separation effect. The experiment was terminated when the chickens were one week old. The body weight of all chickens was determined and caecal contents were collected during post-mortem analysis. In addition, a single sample of feed before (conventional feed) and after (sterile feed) autoclaving was saved for DNA purification and feed microbiota characterisation. To test the viability of bacteria in the conventional feed, an aliquot of the feed was homogenised, serially diluted in PBS, and each dilution was plated on WCHA agar plates. The agar plates were incubated anaerobically at $37^{\circ} \mathrm{C}$ for $48 \mathrm{~h}$ and the bacterial mass was washed with $5 \mathrm{~mL}$ of PBS from the dilution plate with approx. 1000 growing colonies. Washed bacteria were pelleted by centrifugation at $14,000 \times g$ for $1 \mathrm{~min}$ and the pellet was used for DNA purification.

\subsection{Definition of Optimal Age for Administration of Bacterial Strains Intended for Gut Colonisation}

Forty-eight fertilised eggs of ISA Brown line were obtained and assigned to 3 groups with 16 eggs per group. The first group of eggs remained untreated throughout the whole experiment. The second set of 16 eggs was sprayed on day 1 of egg incubation with a mixture of Pseudoflavonifractor capillosus, Anaerotruncus colihominis, Butyricicoccus pullicaecorum, Blautia producta, Clostridium lactatifermentans, Clostridium glycyrrhizinilyticum and Oscillibacter valericigenes. These bacterial species were selected as spore-forming species that might be able to survive as spores on the eggshells [11]. The last group of 16 eggs was treated with the same bacterial mixture on day 1 and the treatment was repeated on day 20 of embryonic development. Eight chickens from control group eggs, nine chickens treated once in the beginning of embryonic development and 12 chickens treated twice during embryonic development were obtained and sacrificed one week after hatching. Caecal contents were collected, DNA from caecal contents was purified and bacteria present in the mixture were quantified by strain-specific real-time PCR (see below).

In the second experiment of this type, Megamonas hypermegale and Bacteroides caecicola were used for spraying eggs and hatching chickens of Ross 308 line. These bacterial strains were selected as capable of efficient colonisation of the chicken caecum [12]. A mixture of these isolates was sprayed over the tray with 50 eggs on day 21 of embryonic development, either at the moment when the first chickens just began to hatch (when less than 10\% of chickens were already hatched), or $10 \mathrm{~h}$ later, when $90 \%$ of chickens were already hatched. Five hatched chickens were taken from each group together with 5 control, non-treated chicks. These chickens were sacrificed when one week old, caecal contents were collected, DNA was purified and the presence of bacteria from the mixture was determined by strain-specific real-time PCR. This experiment was repeated twice.

\subsection{DNA Purification}

All collected samples were stored at $-20{ }^{\circ} \mathrm{C}$ before DNA purification and the storage never exceeded 3 months, to minimise sample degradation. Each sample was homogenised using a MagnaLyser (Roche, Basel, Switzerland) and the homogenate was subjected to DNA purification. QIAmp DNA Stool kit (Qiagen, Hilden, Germany) was used for DNA purification from caecal contents, while DNeasy tissue kit (Qiagen, Hilden, Germany) was used for DNA purification from eggshell or bacterial mass collected from agar plates. Purified DNA was stored at $-20^{\circ} \mathrm{C}$.

\subsection{Quantitative Real-Time PCR}

Strain-specific primers (Table 1) were designed as described previously [13]. Realtime PCR was performed in $3 \mu \mathrm{L}$ volumes in 384-well microplates using QuantiTect SYBR Green PCR Master Mix (Qiagen, Hilden, Germany) and a Nanodrop pipetting station (Innovadyne, Santa Rosa, CA, USA) for dispensing PCR mixtures, as reported elsewhere [13]. 
Table 1. List of primers used for the detection and quantification of target bacterial species.

\begin{tabular}{ccc}
\hline Target Species & Forward Primer & Reverse Primer \\
\hline Pseudoflavonifractor capillosus & GCCAGGAATTTCCCTTCTTC & GGCAGTCTCATGGAGGTAGC \\
Anaerotruncus colihominis & GCAAATGGGCGTATCCTCAA & CTGTTCAATTTCCCGGCAC \\
Butyricicoccus pullicaecorum & CGAGCAGGCAAACGACAA & CCAGGTCTTGGTACCGTCC \\
Blautia producta & GAGAAAAAGGGGCAACAACA & TCAGCATCTTTTCCCCAATC \\
Clostridium lactatifermentans & GGTATCCCCCACGCTTATTT & CCTGCGGTCAATTCTTTGAT \\
Clostridium glycyrrizinilyticum & CTGCAGATAACGCACAGGAA & GTACCGGCAGGCATATCTGT \\
Oscillibacter valericigenes & TGTGAGTCCCAGCTCTACGA & GCAGGGCCTCTCTCCATTTG \\
Megamonas hypermegale & ATTCGCCTTCACGACAATTC & TTACGATATCGGCGAGAACC \\
Bacteroides caecicola & TATACCATGCACGATGAACC & AAATACCTTCTTCCCTCACG \\
\hline
\end{tabular}

2.8. Microbiota Characterisation by Sequencing of V3/V4 Variable Region of $16 S$ rRNA Genes

The concentration of purified DNA was determined spectrophotometrically and DNA samples diluted to $5 \mathrm{ng} / \mathrm{mL}$ were used as a template in PCR with forward primer $5^{\prime}$ TCGTCGGCAGCGTCAGATGTGTATAAGAGACAG-MID-GT-CCTACGGGNGGCWG CAG-3' and reverse primer 5'-GTCTCGTGGGCTCGGAGATGTGTATAAGAGACAG-MIDGT-GACTACHVGGGTATCTAATCC-3'. MIDs represent different sequences of 5, 6, 7, or 9 nucleotides in length, which were used to identify individual samples in the sequencing run. PCR amplification was performed using a KAPA HiFi Hot Start Ready Mix kit (Kapa Biosystems, Woburn, MA, USA) and the resulting PCR products were purified using AMPure beads. Sequencing was performed using MiSeq Reagent Kit v3 (600 cycle) and MiSeq apparatus according to the manufacturer's instructions (Illumina, San Diego, CA, USA), as described previously [6]. The fastq files were imported into QIIME software [14]. Forward and reverse sequences were joined, and chimeric sequences were predicted by the slayer algorithm and excluded from downstream analyses. The non-chimeric sequences were classified by RDP Seqmatch with an operational taxonomic units (OTU) discrimination level set to 97\%. Principal coordinate analysis (PCoA) implemented in QIIME was used for data visualisation.

\subsection{Statistics and Data Availability}

The Mann-Whitney test was used to identify differently abundant taxa among the samples. A $t$-test was used for comparison of body weights of chickens fed either a conventional or a sterile diet. Differences with $p<0.05$ were considered as significant.

\section{Results}

\subsection{Comparison of Eggshell Microbiota and Caecal Microbiota of 7-Day-Old Chicks}

Caecal microbiota of 7-day-old chickens differed from eggshell microbiota (Figure 1A). Microbiota of 7-day-old chickens consisted of Firmicutes and Proteobacteria. On the other hand, eggshell microbiota consisted of representatives of Firmicutes, Bacteroidetes, Actinobacteria and a low representation of Proteobacteria (Figure 1B). The differences were also demonstrated at the OTU level (Figure 1C). When the abundance of OTUs, which formed at least $0.25 \%$ of either caecal or eggshell microbiota, was compared, only two OTUs were present in similar abundance in the microbiota of 7-day-old chickens and eggshells, while 72 OTUs were specific to either type of sample (Figure 1D,E). 
A

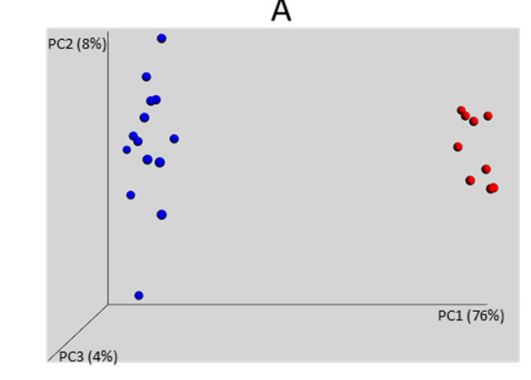

${ }^{5}$ more abundant in the caecal

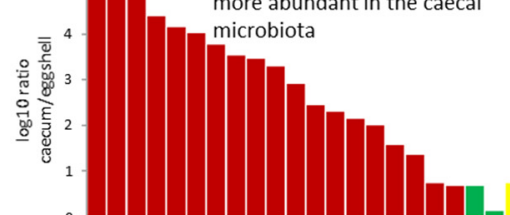

。

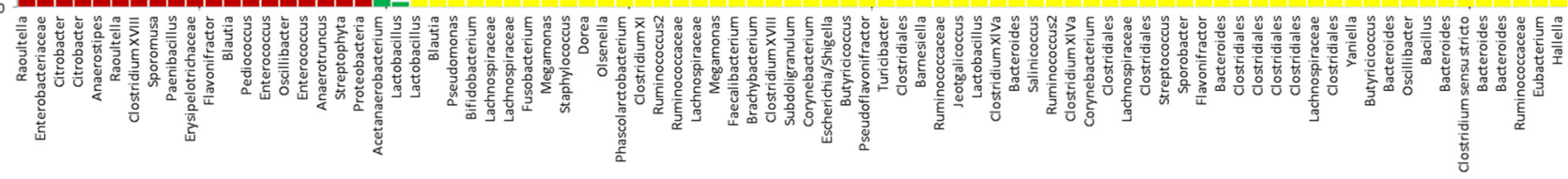

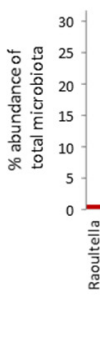

caecal microbiota

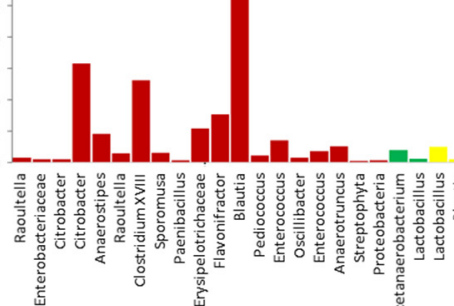

B

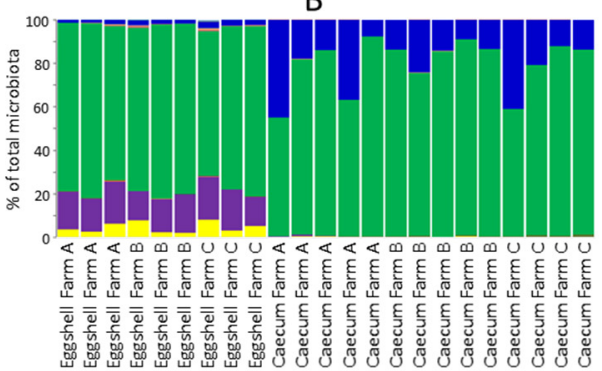

D

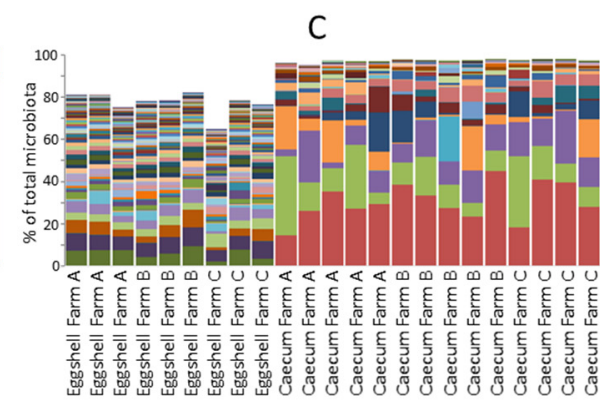

more abundant in eggshell microbiota eggshell microbiota

Figure 1. Comparison of eggshell microbiota and caecal microbiota of one-week old chicks. (A), weighted Principal Coordinate Analysis (PCoA) of caecal microbiota of one-week-old chickens (blue dots) and microbiota of eggshells (red dots). (B), microbiota composition of individual caecal and eggshell samples at phylum level (blue-Proteobacteria, greenFirmicutes, magenta-Bacteroidetes, yellow-Actinobacteria). Microbiota of eggshells resembled caecal microbiota of adult hens due to the presence of Bacteroidetes and Actinobacteria and low representation of Proteobacteria. (C), individual samples at OTU level (top 100 OTUs were used for figure generation) showing completely different composition of caecal microbiota of one-week-old chickens and eggshells microbiota before incubation (see Table S1 for OTU composition). (D), absolute values of $\log _{10}$ ratios of abundances of OTUs forming more than $0.3 \%$ of total microbiota, either in the caecum or in eggshells. Since the biological meaning of the ratios in (D) is influenced also by abundance in of each microbiota member, either in the caecal or eggshell microbiota, these are shown in (E). Abundance in caecal microbiota is shown for the OTUs more abundant in the caecum, and vice versa, abundance in eggshell microbiota is shown for the OTUs more abundant in eggshell microbiota.

The caecal microbiome of 7-day-old chickens was characteristic for this particular age $[1,4,5]$. Eggshell microbiota was a mixture of adult hen microbiota and environmental microbiota (Table S1). Eggshell microbiota was dominated by microbiota deposited by the hen during egg laying, since Faecalibacterium prausnitzii and Oscillibacter ruminantium formed $7.20 \%$ and $5.98 \%$ of total eggshell microbiota, respectively. In addition, different Bacteroides species formed $12 \%$ of total microbiota, and Megamonas hypermegale and M. funiformis formed $1.20 \%$ and $1.94 \%$ of all eggshell microbiota, respectively. These are all microbiota members characteristic for adult hens. The third most abundant OTU of eggshell microbiota was represented by Clostridium sporogenes forming $4.79 \%$ of eggshell microbiota. In addition, Actinomycetales (genera Corynebacterium, Brachybacterium or Yaniella) formed $1.40 \%$ of eggshell microbiota, indicating the environment as a source $[15,16]$. Bacillus coagulans formed 2.13\% of total eggshell microbiota and Staphylococcaceae (genera 
Jeotgalicoccus, Salinicoccus and Staphylococcus) formed 1.09\% of eggshell microbiota, further showing the contribution of the environment [17-20].

\subsection{Gut Microbiota of 7-Day-Old Chickens Originating from Different Parent Flocks}

Next, we analysed the microbiota composition in the caeca of chickens originating from three different parent flocks, which were hatched and raised together during the first week of life. PCoA showed that all the chickens were randomly distributed and there was no clustering according to origin of the reproductive parent flock (Figure 2). The chickens were, therefore, colonised from environmental sources and not by the microbiota of their (different) parents.

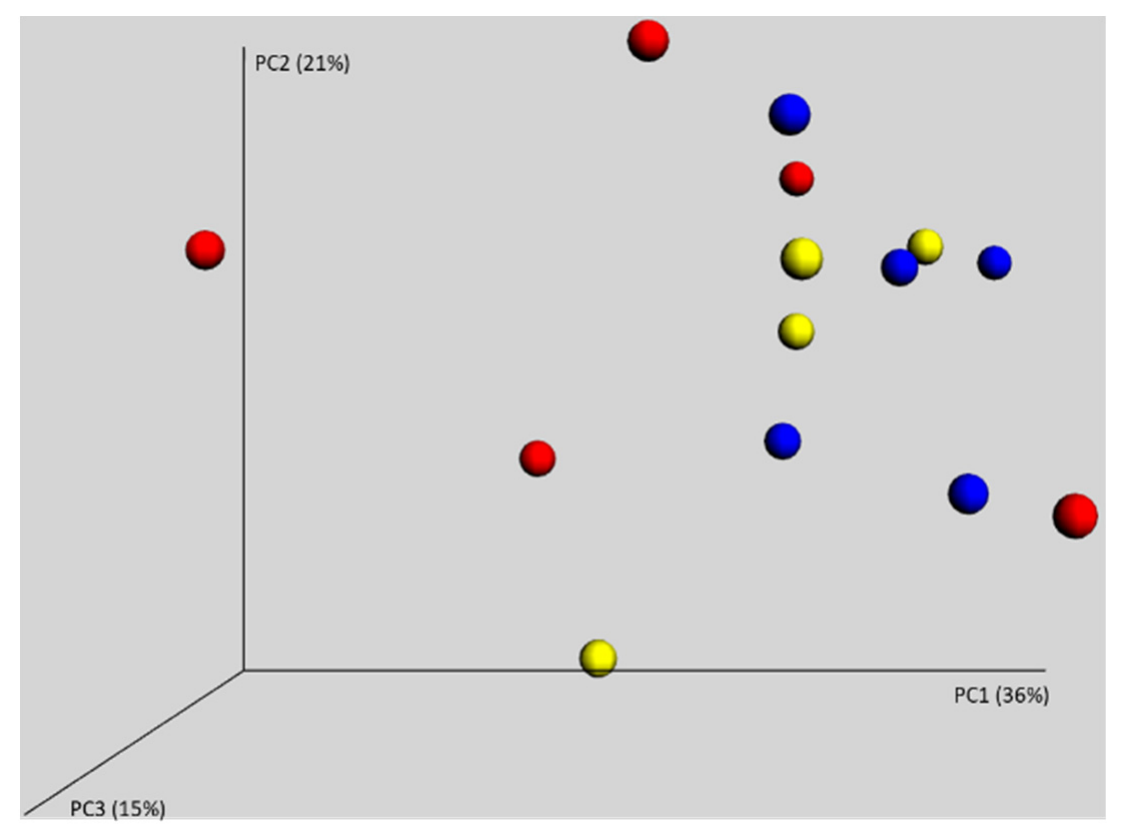

Figure 2. Weighted PCoA of caecal samples of chickens from three different parent flocks. No clustering according to origin of chickens defined by different colours was observed. Parents, therefore, did not play any role in the establishment of gut microbiota in the chickens in commercial production.

\subsection{Feed as a Source of Gut Microbiota}

Next, we tested the role of feed in the development of caecal microbiota during the first week of life. Two groups of chickens were provided with either autoclaved feed (sterile feed) or the same feed without autoclaving (conventional feed). Caecal microbiota of both groups of chickens was similar and differed from feed microbiota (Figure 3A). Characterisation of feed microbiota was complicated by the fact that sequences corresponding to plant chloroplast rRNA formed $59.1 \%$ of all sequences obtained for the conventional feed sample (Figure 3B). In sterile feed, the DNA including the chloroplast DNA was degraded; it was impossible to purify any DNA from this type of sample (Figure 3C) and obtain 16S rRNA amplification products (Figure 3D). Consequently, the number of reads obtained for the sterile feed sample was extremely low (Figure 3E) and OTU composition in sterile feed must be considered with great care. 

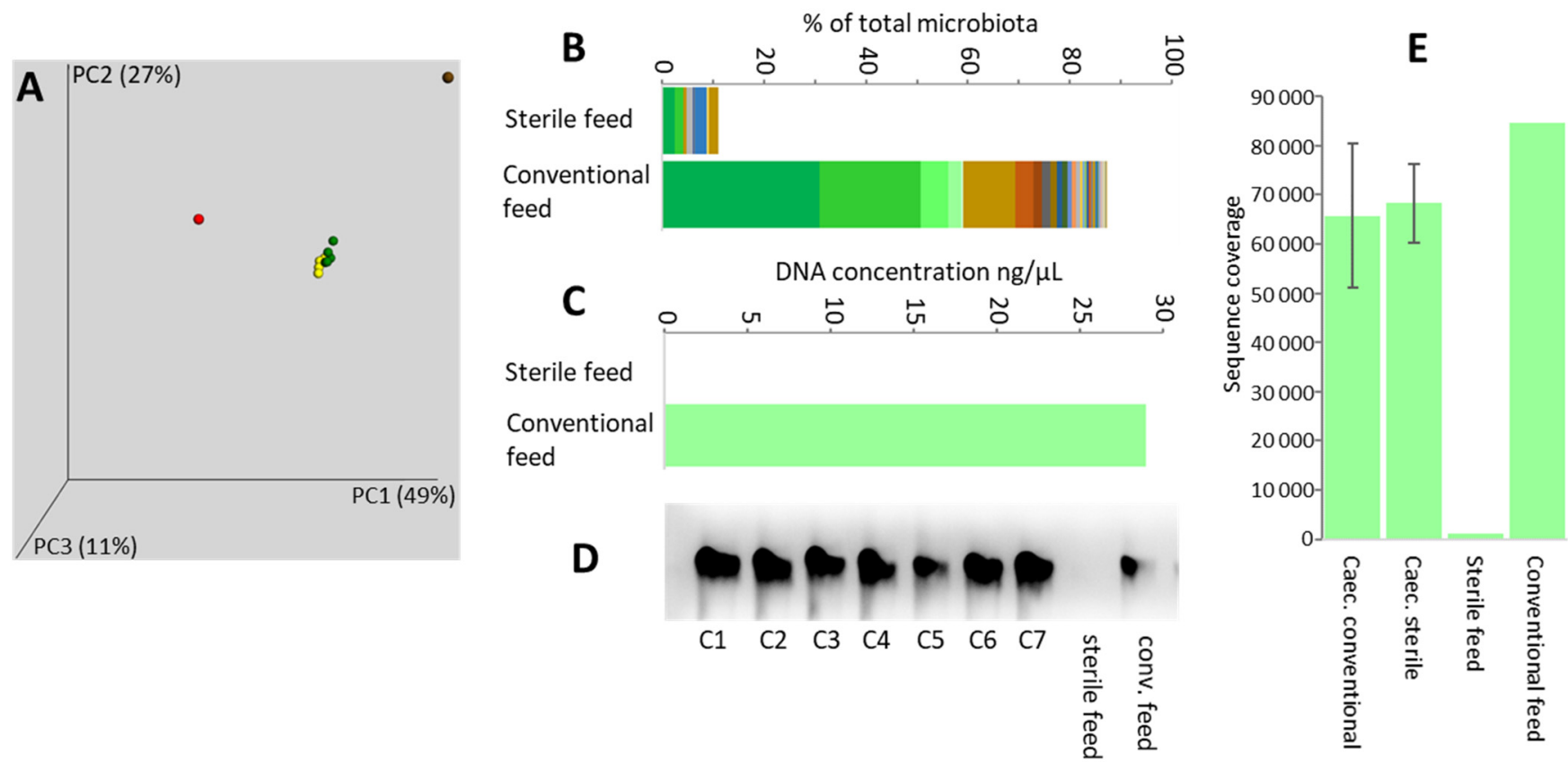

Figure 3. Feed as a source of chicken gut microbiota. (A), weighted PCoA analysis of microbiota in conventional (brown dot) and sterile feed (red dot) differed from the caecal microbiota of one-week-old chickens fed with conventional (green dots) or sterile feed (yellow dots). Conventional feed microbiome was dominated by chloroplast rRNA sequences from plants used for feed manufacturing. Top 30 OTUs from conventional feed showed in (B) formed $87.3 \%$ of all reads, and chloroplast assigned reads (shown in shades of green) formed 59.1\% of all reads (see Table S2 for taxa composition). DNA in feed samples was degraded by autoclaving and low DNA concentration (C), low quality of PCR products ((D), c1-c7 stands for 7 caecal samples, followed by amplification products obtained using DNA from sterile or conventional feed as template) and low number of sequences (E) were obtained for the sterile feed sample.

Microbiota of chickens fed a conventional or sterile diet, despite qualitative similarities, exhibited quantitative differences at the OTU level. Whether chickens were fed with conventional or sterile feed, the top 15 OTUs represent more than $90 \%$ of total microbiota for both groups. Out of these, Anaerostipes caccae was significantly more abundant in the microbiota of chickens fed conventional feed, while Flavonifractor plautii, Enterococcus hirae and Clostridium paraputrificum were more abundant in the microbiota of chickens fed sterile feed (Figure 4A). The same OTUs represented minor fractions in conventional feed microbiota and in the washes from anaerobically incubated WCHA agar plates inoculated with serially diluted homogenates of conventional feed. Similarly, OTUs common in the conventional feed (Pantoea vagans, Curtobacterium flaccumfaciens, Erwinia aphidicola, Pseudomonas trivialis, Stenotrophomonas rhizophila, Sphingomonas olei) or in bacterial pools washed from agar plates after anaerobic culture (Bacillus licheniformis) did not efficiently colonise the chicken caecum (Figure 4B). All results suggest that feed did not act as the major source of gut microbiota, although autoclaving of feed affected its nutritional values and allowed for a different microbiota composition to develop in the chickens. The effect of autoclaving feed was confirmed by the lower body weight of chickens provided with the autoclaved feed in comparison to those fed conventional feed (Figure 4C).

\subsection{Definition of an Optimal Time Point for Administration of Bacterial Strains during Hatching}

Finally, we attempted to fine the timepoint during prenatal or neonatal development at which it is possible to efficiently colonise chickens. A mixture of seven different sporeforming species was sprayed over the eggs on day 1 of embryonic development, or both on day 1 and day 20 of embryonic development. When the caecal microbiota composition was determined in one-week-old chicks, none of the strains used for egg treatment were detected in the chicken caecum (Table S3). Next, we used an additional two strains, Megamonas hypermegale and Bacteroides caecicola, which efficiently colonised the chicken 
caecum [12]. A mixture of these two isolates was sprayed over the box with incubated eggs on day 21 of embryonic development, either at the moment when less than $10 \%$ of chickens were already hatched, or approximately $10 \mathrm{~h}$ later, when $90 \%$ of chickens were hatched. No colonisation was recorded when the boxes with eggs and a few already hatched chickens were treated. In contrast, successful colonisation of all tested chickens was observed when the tray was sprayed when $90 \%$ of chickens were already hatched (Figure 5).

A

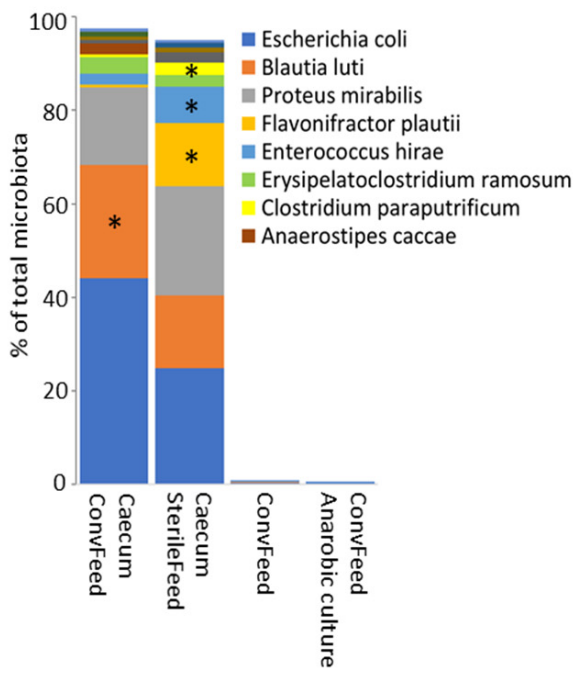

B

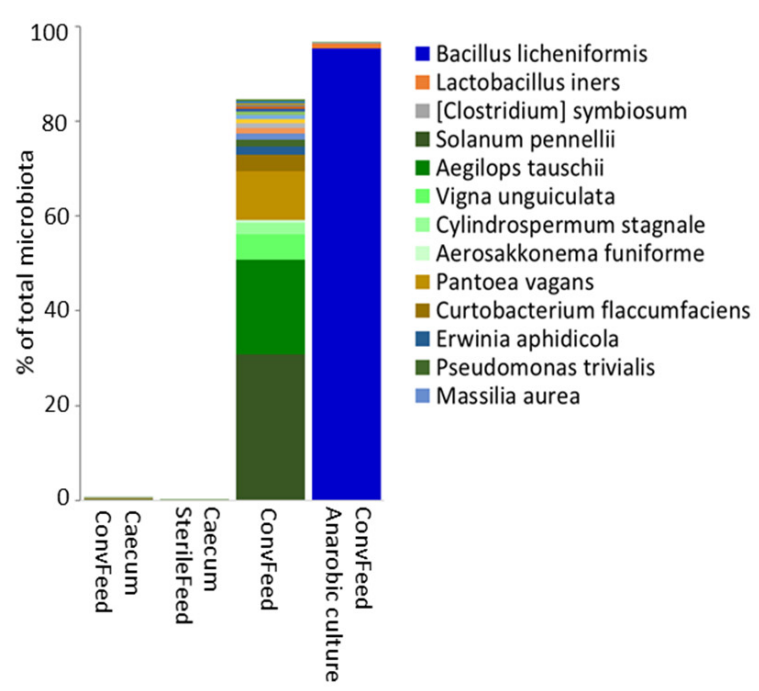

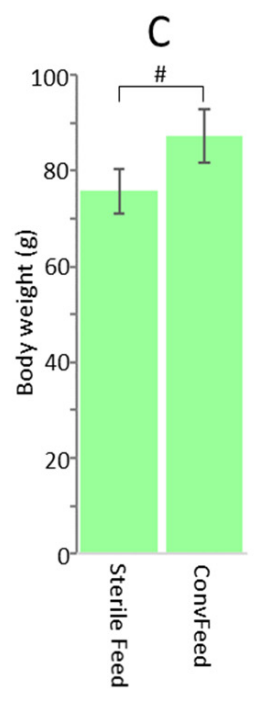

Figure 4. Influence of conventional and sterile feed on caecal microbiota composition and body weight of chickens. (A), Composition of caecal microbiota in the chickens was affected by conventional or sterile feed. Asterisks indicate OTUs differently abundant in microbiota of chickens fed conventional or sterile feed. (B), Feed microbiota consisted of chloroplast $16 \mathrm{~S}$ rRNA (different shades of green in conventional feed) and the only viable bacterial species in the feed was Bacillus licheniformis. Feed, therefore, was not the main source of gut microbiota for chickens. Autoclaving resulted in degradation of plant chloroplast DNA and negatively affected nutritional quality, which influenced microbiota composition (A) but also chicken body weight (C). *-significantly different in microbiota of chickens provided with conventional or sterile feed by Mann-Whitney test. \#-significantly different by $t$-test.

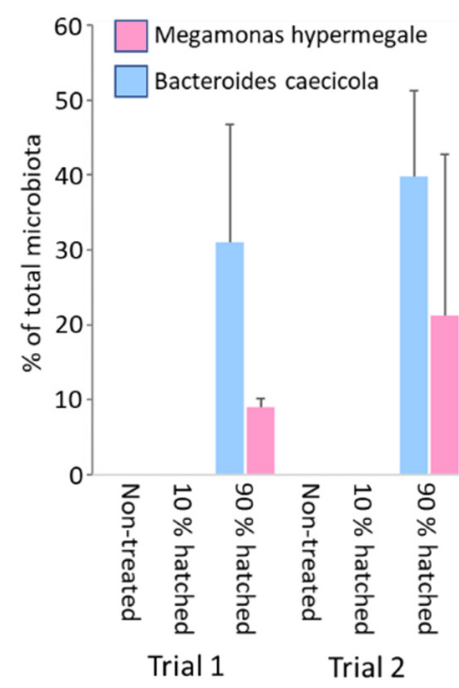

Figure 5. Definition of the timepoint when chickens can be colonised by experimentally administered bacterial strains. Hatching eggs were sprayed with a mixture of Bacteroides caecicola and Megamonas hypermegale, either when $10 \%$ of chickens were already hatched or when $90 \%$ of chickens were hatched. Five control chickens were not treated at all. All tested chickens became efficiently colonised only when the majority of them were already hatched and became active. 


\section{Discussion}

In this study, we addressed the origin of microbiota that appears in the chicken caecum shortly after hatching. Although hens deposit their microbiota on eggshells and adult-type microbiota was found on the eggshells [1,21-23], this was not vertically transmitted. The fact that parents do not act as a source of gut microbiota for newly hatched chickens in commercial production was also confirmed by hatching chickens from eggs originating from different parent flocks. Samples from these chickens did not form separate clusters, their microbiota was similar, and these chickens, therefore, had to be colonised from the same environmental sources after hatching. The fact that transmission via eggshell deposition is not efficient was confirmed also by experimental deposition of spore-forming Clostridiales onto the eggshell, either at the beginning of egg incubation or a day before hatching.

The eggshells were contaminated by Actinomycetales and Staphylococcaceae. These bacteria are common in chicken litter $[15,16,24]$ or in the air of poultry houses $[18,25]$ and are known from previous reports to contribute to eggshell microbiota [26-28]. Whether viable or not, these were definitively not transferred to the chicken intestinal tract. Feed and drinking water also did not act as a major source of gut microbiota. There were variations in quantities of different OTUs in chickens fed conventional and sterile feed, but qualitatively the same major OTUs were present in microbiota of both groups of chicks. When checking for viable anaerobic bacteria in conventional feed, Bacillus licheniformis dominated, followed by Lactobacillus iners and Clostridium symbiosum, but none of these bacteria colonised the chicken intestinal tract. The second most common OTU in the caeca of one-week-old chicks, Blautia luti, formed $0.16 \%$ of total feed microbiota, which does not exclude its feed origin. However, $\mathrm{Bl}$. luti did not grow after anaerobic culture of feed samples, which suggests that it was present in the feed in inactivated form. Moreover, the eight most abundant anaerobes from the caeca of chicks, Flavonifractor plautii, Erysipelatoclostridium ramosum, Clostridium paraputrificum, Anaerostipes caccae, Clostridium perfringens, Clostridium saccharogumia, Pediococcus acidilactici and Romboutsia timonensis, each formed $0.02 \%$ or less of the total feed microbiota, and except for Romboutsia timonensis, none of these OTUs were detected in washes from anaerobic cultures. Feed, therefore, does not represent a major source of gut microbiota for chickens in commercial production although feed shapes gut microbiota composition, as observed in the chickens fed with conventional and sterilised feed.

Finally, we tested at what time during the hatching period it is possible to administer gut microbiota and achieve successful colonisation. Spraying of eggs or even chickens at the very beginning of the hatching process, when the majority of chickens were still in eggs, did not result in efficient colonisation. This also means that spraying of eggs any time during incubation with strict anaerobes, i.e., before the hatching, will not result in efficient colonisation. Chickens could be colonised only when already hatched, not considering in ovo inoculation with facultative anaerobes $[29,30]$. The early administration of probiotics, therefore, results in treatment failure, while delayed treatment of chickens with complex microbiota is also not recommended since such products do not act therapeutically [7]. Therefore, the window for reasonable administration is quite short, lasting for a few hours at hatching, before placement on a farm where chickens may become colonised by microbiota of unintended composition. Although this window is rather short, it is still long enough to logistically arrange the whole production process and include probiotic treatment at an appropriate time and place.

Supplementary Materials: The following are available online at https:/ / www.mdpi.com/article/10 .3390 / microorganisms 9071480 / s1, Table S1. OTU table of caecal and eggshell microbiota. Table S2. OTU table of feed microbiota and chicken caecum microbiota fed conventional or sterile feed. Table S3. Real-time PCR quantification of 7 strains from phylum Firmicutes used for spraying of eggs on day 1 and/or day 20 of embryonic development. 
Author Contributions: J.V., A.S. and M.F. (Marcela Faldynova) was responsible for the experiments focused on eggshell microbiota. D.K., M.C., Z.S. and T.K., M.Z., performed 16S rRNA sequencing and data analysis. H.J. and J.M. prepared bacterial cultures for all the experiments. M.F. (Marian Foltyn) and Z.T. were responsible for spraying hatching eggs and chickens in hatchery. I.R. designed the study and wrote initial draft of the manuscript. All authors have read and agreed to the published version of the manuscript.

Funding: This work has been supported by project RVO0518 of the Czech Ministry of Agriculture and INPOMED ATCZ194 project from the Czech Ministry of Regional Development.

Institutional Review Board Statement: The handling of animals was performed in accordance with current Czech legisla-tion (Animal Protection and Welfare Act No. 246/1992 Coll. of the Government of the Czech Republic). The specific experiments were approved by the Ethics Committee of the Veterinary Research Institute followed by the Committee for Animal Welfare of the Ministry of Agriculture of the Czech Republic (permit number MZe1922).

Informed Consent Statement: Not applicable.

Data Availability Statement: The generated raw sequence dataset is available and was deposited in the NCBI Short Read Archive database under BioProject accession number PRJNA718522. All remaining data generated or analysed during this study are included in this published article and its supplementary information files.

Acknowledgments: Authors wish to thank Peter Eggenhuizen for English language corrections.

Conflicts of Interest: The author declares no conflict of interest.

\section{References}

1. Videnska, P.; Sedlar, K.; Lukac, M.; Faldynova, M.; Gerzova, L.; Cejkova, D.; Sisak, F.; Rychlik, I. Succession and Replacement of Bacterial Populations in the Caecum of Egg Laying Hens over Their Whole Life. PLoS ONE 2014, 9, e115142. [CrossRef]

2. Gao, P.; Ma, C.; Sun, Z.; Wang, L.; Huang, S.; Su, X.; Xu, J.; Zhang, H. Feed-additive probiotics accelerate yet antibiotics delay intestinal microbiota maturation in broiler chicken. Microbiome 2017, 5, 1-14. [CrossRef] [PubMed]

3. Xi, Y.; Shuling, N.; Kunyuan, T.; Qiuyang, Z.; Hewen, D.; ChenCheng, G.; Tianhe, Y.; Liancheng, L.; Xin, F. Characteristics of the intestinal flora of specific pathogen free chickens with age. Microb. Pathog. 2019, 132, 325-334. [CrossRef]

4. Stanley, D.; Geier, M.S.; Hughes, R.J.; Denman, S.; Moore, R.J. Highly Variable Microbiota Development in the Chicken Gastrointestinal Tract. PLoS ONE 2013, 8, e84290. [CrossRef] [PubMed]

5. Ranjitkar, S.; Lawley, B.; Tannock, G.; Engberg, R.M. Bacterial Succession in the Broiler Gastrointestinal Tract. Appl. Environ. Microbiol. 2016, 82, 2399-2410. [CrossRef] [PubMed]

6. Kubasova, T.; Kollarcikova, M.; Crhanova, M.; Karasova, D.; Cejkova, D.; Sebkova, A.; Matiasovicova, J.; Faldynova, M.; Pokorna, A.; Cizek, A.; et al. Contact with adult hen affects development of caecal microbiota in newly hatched chicks. PLoS ONE 2019, 14, e0212446. [CrossRef] [PubMed]

7. Varmuzova, K.; Kubasova, T.; Davidova-Gerzova, L.; Sisak, F.; Havlickova, H.; Sebkova, A.; Faldynova, M.; Rychlik, I. Composition of Gut Microbiota Influences Resistance of Newly Hatched Chickens to Salmonella Enteritidis Infection. Front. Microbiol. 2016, 7, 957. [CrossRef] [PubMed]

8. Rantala, M.; Nurmi, E. Prevention of the growth ofSalmonella infantisin chicks by the flora of the alimentary tract of chickens. Br. Poult. Sci. 1973, 14, 627-630. [CrossRef]

9. Stanley, D.; Hughes, R.J.; Geier, M.S.; Moore, R.J. Bacteria within the Gastrointestinal Tract Microbiota Correlated with Improved Growth and Feed Conversion: Challenges Presented for the Identification of Performance Enhancing Probiotic Bacteria. Front. Microbiol. 2016, 7, 187. [CrossRef]

10. Rikimaru, K.; Takahashi, H. A simple and efficient method for extraction of PCR-amplifiable DNA from chicken eggshells. Anim. Sci. J. 2009, 80, 220-223. [CrossRef]

11. Medvecky, M.; Cejkova, D.; Polansky, O.; Karasova, D.; Kubasova, T.; Cizek, A.; Rychlik, I. Whole genome sequencing and function prediction of 133 gut anaerobes isolated from chicken caecum in pure cultures. BMC Genom. 2018, 19, 1-15. [CrossRef]

12. Kubasova, T.; Kollarcikova, M.; Crhanova, M.; Karasova, D.; Cejkova, D.; Sebkova, A.; Matiasovicova, J.; Faldynova, M.; Sisak, F.; Babak, V.; et al. Gut Anaerobes Capable of Chicken Caecum Colonisation. Microorganisms 2019, 7, 597. [CrossRef]

13. Kollarcikova, M.; Faldynova, M.; Matiasovicova, J.; Jahodarova, E.; Kubasova, T.; Seidlerova, Z.; Babak, V.; Videnska, P.; Cizek, A.; Rychlik, I. Different Bacteroides Species Colonise Human and Chicken Intestinal Tract. Microorganisms 2020, 8, 1483. [CrossRef] [PubMed]

14. Caporaso, J.G.; Kuczynski, J.; Stombaugh, J.; Bittinger, K.; Bushman, F.D.; Costello, E.K.; Fierer, N.; Peña, A.G.; Goodrich, J.K.; Gordon, J.I.; et al. QIIME Allows Analysis of High-Throughput Community Sequencing data. Nat. Methods 2010, 7, 335-336. [CrossRef] [PubMed] 
15. Johnson, T.J.; Youmans, B.P.; Noll, S.; Cardona, C.; Evans, N.P.; Karnezos, T.P.; Ngunjiri, J.M.; Abundo, M.C.; Lee, C.-W. A Consistent and Predictable Commercial Broiler Chicken Bacterial Microbiota in Antibiotic-Free Production Displays Strong Correlations with Performance. Appl. Environ. Microbiol. 2018, 84, e00362-18. [CrossRef] [PubMed]

16. Bucher, M.G.; Zwirzitz, B.; Oladeinde, A.; Cook, K.; Plymel, C.; Zock, G.; Lakin, S.; Aggrey, S.E.; Ritz, C.; Looft, T.; et al. Reused poultry litter microbiome with competitive exclusion potential against Salmonella Heidelberg. J. Environ. Qual. 2020, 49, 869-881. [CrossRef]

17. De Cesare, A.; Caselli, E.; Lucchi, A.; Sala, C.; Parisi, A.; Manfreda, G.; Mazzacane, S. Impact of a probiotic-based cleaning product on the microbiological profile of broiler litters and chicken caeca microbiota. Poult. Sci. 2019, 98, 3602-3610. [CrossRef] [PubMed]

18. Martín, E.; Fallschissel, K.; Kampfer, P.; Jäckel, U. Detection of Jeotgalicoccus spp. in poultry house air. Syst. Appl. Microbiol. 2010, 33, 188-192. [CrossRef]

19. Cressman, M.D.; Yu, Z.; Nelson, M.C.; Moeller, S.J.; Lilburn, M.S.; Zerby, H.N. Interrelations between the Microbiotas in the Litter and in the Intestines of Commercial Broiler Chickens. Appl. Environ. Microbiol. 2010, 76, 6572-6582. [CrossRef] [PubMed]

20. Adkins, P.; Dufour, S.; Spain, J.; Calcutt, M.; Reilly, T.; Stewart, G.; Middleton, J. Cross-sectional study to identify staphylococcal species isolated from teat and inguinal skin of different-aged dairy heifers. J. Dairy Sci. 2018, 101, 3213-3225. [CrossRef]

21. Callaway, T.R.; Dowd, S.; Wolcott, R.D.; Sun, Y.; McReynolds, J.L.; Edrington, T.S.; Byrd, J.A.; Anderson, R.C.; Krueger, N.; Nisbet, D.J. Evaluation of the bacterial diversity in cecal contents of laying hens fed various molting diets by using bacterial tag-encoded FLX amplicon pyrosequencing. Poult. Sci. 2009, 88, 298-302. [CrossRef] [PubMed]

22. Nordentoft, S.; Mølbak, L.; Bjerrum, L.; De Vylder, J.; Van Immerseel, F.; Pedersen, K. The influence of the cage system and colonisation of Salmonella Enteritidis on the microbial gut flora of laying hens studied by T-RFLP and 454 pyrosequencing. BMC Microbiol. 2011, 11, 187. [CrossRef] [PubMed]

23. Videnska, P.; Rahman, M.; Faldynova, M.; Babak, V.; Matulova, M.E.; Prukner-Radovcic, E.; Krizek, I.; Smole-Mozina, S.; Kovac, J.; Szmolka, A.; et al. Characterization of Egg Laying Hen and Broiler Fecal Microbiota in Poultry Farms in Croatia, Czech Republic, Hungary and Slovenia. PLoS ONE 2014, 9, e110076. [CrossRef] [PubMed]

24. Wang, L.; Lilburn, M.; Yu, Z. Intestinal Microbiota of Broiler Chickens As Affected by Litter Management Regimens. Front. Microbiol. 2016, 7, 593. [CrossRef]

25. Oppliger, A.; Charrière, N.; Droz, P.-O.; Rinsoz, T. Exposure to Bioaerosols in Poultry Houses at Different Stages of Fattening; Use of Real-time PCR for Airborne Bacterial Quantification. Ann. Occup. Hyg. 2008, 52, 405-412. [CrossRef]

26. Olsen, R.; Kudirkiene, E.; Thøfner, I.; Pors, S.; Karlskov-Mortensen, P.; Li, L.; Papasolomontos, S.; Angastiniotou, C.; Christensen, J. Impact of egg disinfection of hatching eggs on the eggshell microbiome and bacterial load. Poult. Sci. 2017, 96, 3901-3911. [CrossRef] [PubMed]

27. Trudeau, S.; Thibodeau, A.; Côté, J.-C.; Gaucher, M.-L.; Fravalo, P. Contribution of the Broiler Breeders' Fecal Microbiota to the Establishment of the Eggshell Microbiota. Front. Microbiol. 2020, 11, 666. [CrossRef]

28. Maki, J.J.; Bobeck, E.A.; Sylte, M.J.; Looft, T. Eggshell and environmental bacteria contribute to the intestinal microbiota of growing chickens. J. Anim. Sci. Biotechnol. 2020, 11, 1-17. [CrossRef]

29. Wilson, K.M.; Rodrigues, D.; Briggs, W.N.; Duff, A.F.; Chasser, K.M.; Bielke, L.R. Evaluation of the impact of in ovo administered bacteria on microbiome of chicks through 10 days of age. Poult. Sci. 2019, 98, 5949-5960. [CrossRef]

30. de Oliveira, J.E.; van der Hoeven-Hangoor, E.; van de Linde, I.B.; Montijn, R.C.; van der Vossen, J.M.B.M. In ovo inoculation of chicken embryos with probiotic bacteria and its effect on posthatch Salmonella susceptibility. Poult. Sci. 2014, 93, 818-829. [CrossRef] 